\section{Surgical treatment for primary lymphoma of the heart simulating prosthetic mitral valve thrombosis}

\section{To the Editor:}

Primary malignant lymphomas involving only the heart are rare tumors. They are rarely diagnosed ante mortem and produce a variety of clinical manifestations. Surgical removal of this rare tumor has been reported only one time. ${ }^{1}$ We present a new case of resection of primary cardiac lymphoma, which simulated prosthetic mitral valve thrombosis in a 66-year-old woman.

A 66-year-old woman was admitted to the intensive care unit for pulmonary edema after a history of syncope. She had had mitral valve replacement 8 years earlier (St. Jude Medical heart valve $25 \mathrm{M}$, St. Jude Medical, Inc., St. Paul, Minn.). Transthoracic and transesophageal echography showed a large, mobile left atrial mass (Fig. 1). The patient was taken to the operating room with the diagnosis of valve thrombosis.

The patient was operated on with total cardiopulmonary bypass, moderate hypothermia, and cardioplegia. The left atrium was opened with a long incision in the interatrial groove. No thrombus was found, but a pedicled, polypoid, gray-white tumor was attached to the atrial wall close to the mitral anulus. It was excised with the attachment of the pedicle.

Pathologic examination revealed a 4 by 2.5 by $2 \mathrm{~cm}$ firm mass, histologically a large-cell lymphoma (Fig. 2), without myocardium infiltration. The postoperative course was uneventful. Bone biopsy did not show lymphomatous involvement. The computed tomographic scans of the abdomen, thorax, pelvis, and head showed no abnormalities. She did not receive chemotherapy and was symptom free 6 months later, with normal findings on transesophageal echography (Fig. 3).

Primary lymphoma of the heart is a rare disorder with a poor prognosis. ${ }^{2}$ In most of the reported cases the diagnosis was made at autopsy or the patient died shortly after diagnosis despite therapy. ${ }^{3}$ In all reported cases of lymphoma of the heart, the

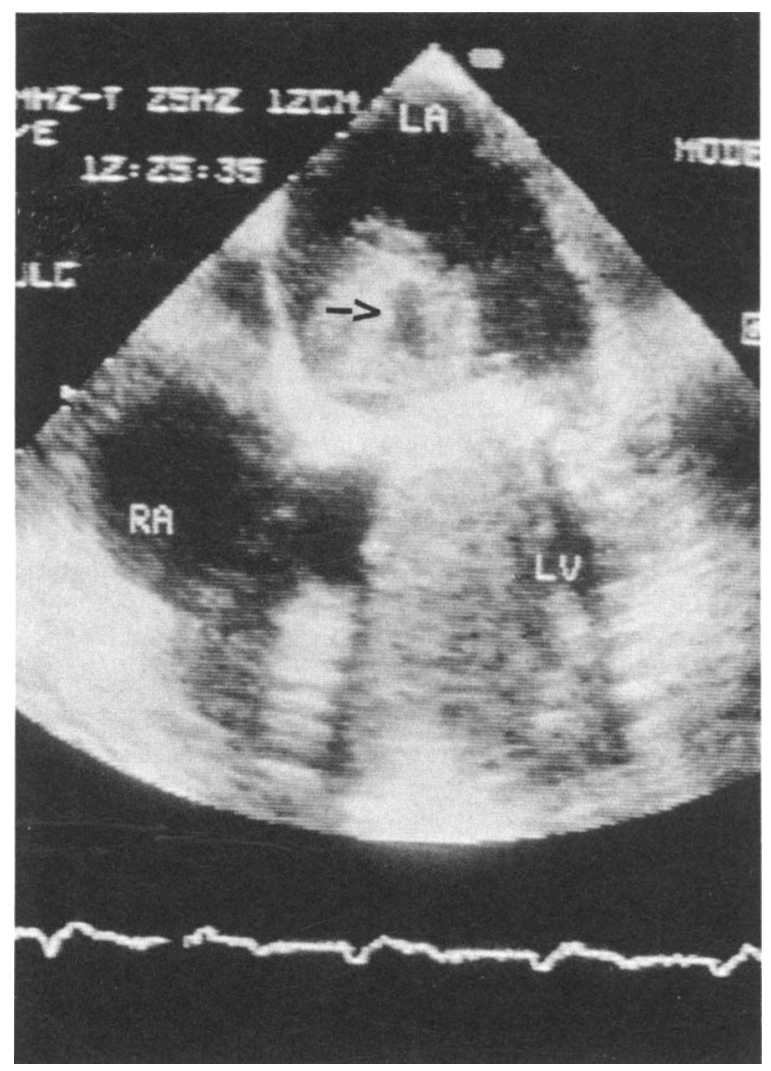

Fig. 1. Transesophageal two-dimensional echocardiographic image showing a left atrial mass (arrow). $R A$, Right atrium; $L A$, left atrium; $L V$, left ventricle.

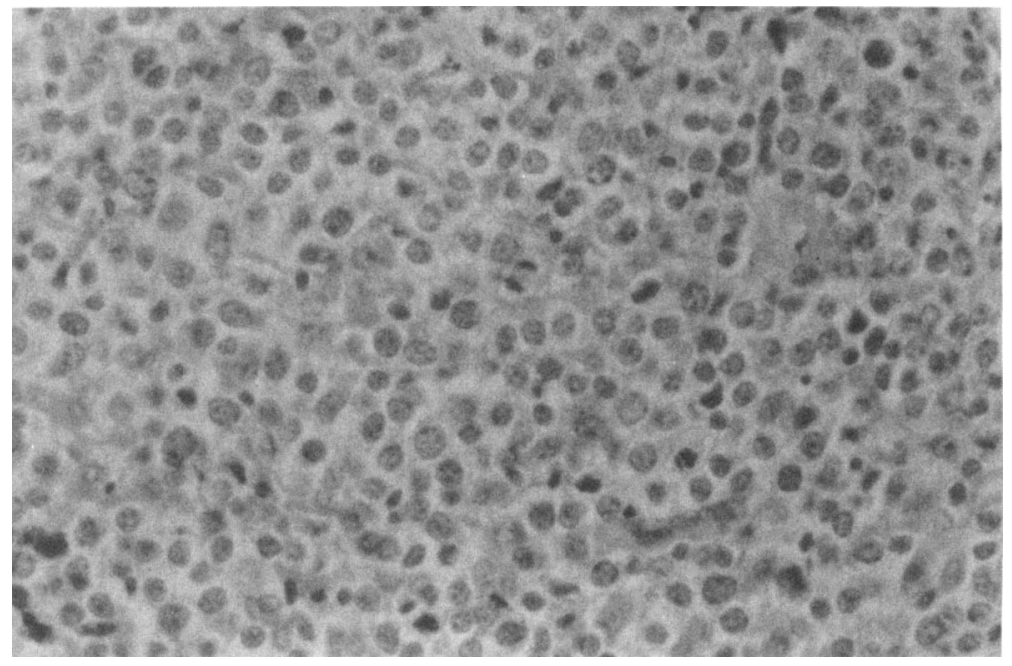

Fig. 2. Microscopic section showing a large-cell lymphoma. 


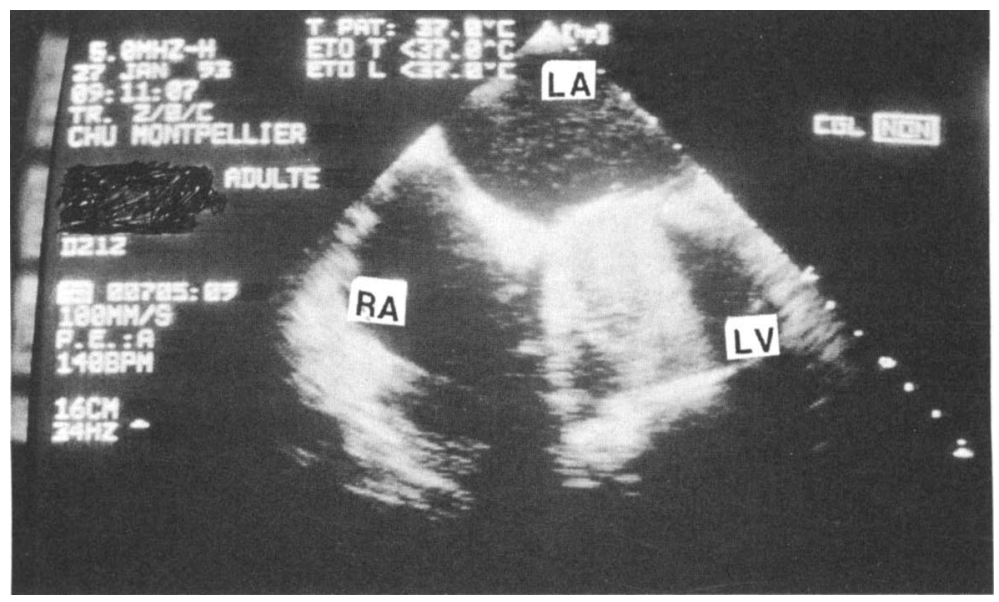

Fig. 3. Transesophageal two-dimensional echocardiographic follow-up study 6 months after the operation. $R A$, Right atrium; $L A$, left atrium; $L V$, left ventricle.

tumor involved a large part of the cardiac wall and treatment consisted of biopsy and chemotherapy. Despite this treatment, the patient usually died within 2 to 3 weeks except in the case reported by Nand and associates, ${ }^{4}$ whose patient was alive 18 months after diagnosis and aggressive chemotherapy.

The only case of surgical resection for malignant lymphoma of the heart invading the right atrial wall and both venae cavae was reported by Takagi and associates.' A chemotherapy regimen was instituted in the postoperative period and the patient died of acute pneumonia 234 days after the operation. In our case, the primary lymphoma of the heart was limited and totally excised. Because the patient had a mitral valve prosthesis, chemotherapy seemed to us too aggressive. Regular clinical and transesophageal echographic assessment seems sufficient to detect recurrence. Six months after the operation, the patient was symptom free.

B. Albat, $M D$

P. Messner-Pellenc, $M D$

A. Thévenet, $M D$

Department of Cardiovascular Surgery and Cardiology University Hospital Montpellier, France

\section{REFEREN CES}

1. Takagi M, Kugimiya T, Fujii T, et al. Extensivesurgery for primary malignant lymphoma of the heart. J Cardiovasc Surg 1992;33:570-2.

2. Curtsinger CR, Wilson MJ, Yoneda K. Primary cardiac lymphoma. Cancer 1989;64:521-5.

3. Chou ST, Arkles LB, Gill GD, et al. Primary lymphoma of the heart: a case report. Cancer 1983;52:744-7.

4. Nand S, Mullen GM, Lonchyna VA, Moncada R. Primary lymphoma of the heart: prolonged survival with early systemic therapy in a patient. Cancer 1991;60:2289-92.

\section{Postoperative diaphragmatic hernia after use of the right gastroepiploic artery for coronary artery bypass grafting}

To the Editor:

Along with the internal thoracic arteries, the right gastroepiploic artery is now an established arterial graft for myocardial revascularization, especially in the setting of reoperation or when no suitable vein is available., ${ }^{1,2}$ This type of operation is seldom associated with an increased risk for postoperative complications other than those of standard coronary artery bypass grafting. ${ }^{3}$ We describe the case history of a patient who had an incarcerated postoperative diaphragmatic hernia after myocardial revascularization with the right gastroepiploic artery graft, a complication specific only for this type of coronary artery bypass graft.

A-56-year-old man with severe three-vessel coronary artery disease and New York Heart Association class III angina pectoris, as well as a history of bronchial asthma, underwent uneventful coronary artery bypass grafting. Because only a single $30 \mathrm{~cm}$ long segment of the great saphenous vein was available because of previous vein stripping, we used two arterial conduits for myocardial revascularization, the left internal thoracic artery and the right gastroepiploic artery. The patient received five distal anastomoses: the left internal thoracic artery was implanted into the left anterior descending artery, the vein segment was used for revascularization of the first and second obtuse marginal branches of the left circumflex artery and for the diagonal branch, and the right gastroepiploic artery was implanted into the right posterior descending artery. The pedicle of the right gastroepiploic artery was brought into the pericardial sac through a hole in the diaphragm, passing the stomach and the liver posteriorly. The postoperative course was complicated by instability of the sternum because of several episodes of cough associated with acute exacerbation of asthma, and the sternum was refixed 3 weeks after the coronary artery operation. Neither clinical nor laboratory examinations 